\title{
Second International Workshop On the Evaluation of Collaborative Information Seeking and Retrieval (Ecol'17)
}

\author{
Leif Azzopardi \\ University of Strathclyde \\ Glasgow, UK \\ Leif.Azzopardi@strath.ac.uk
}

\author{
Jeremy Pickens \\ Catalyst Repository Systems \\ Palo Alto, CA, USA \\ jpickens@catalystsecure.com
}

\author{
Chirag Shah \\ Rutgers University \\ New Brunswick, NJ, USA \\ chirags@rutgers.edu
}

\author{
Laure Soulier \\ Sorbonne Universités, UPMC \\ Univ Paris 06, LIP6 UMR 7606 \\ 75005 Paris, France \\ laure.soulier@lip6.fr
}

\author{
Lynda Tamine \\ Université de Toulouse UPS IRIT \\ Toulouse, France \\ tamine@irit.fr
}

\begin{abstract}
The workshop on the evaluation of collaborative information retrieval and seeking (ECol) is held in conjunction with the ACM SIGIR Conference on Human Information Interaction \& Retrieval (CHIIR) in Oslo, Norway. To make the workshop active and the participant pro-active, we released datasets and tools so as to help researchers contributing to the formalization of evaluation frameworks for challenging collaborative tasks. The workshop is split into two parts. First, a presentation session. Then, the afternoon is devoted to group discussion addressing challenges of evaluating and designing models for social and collaborative search.
\end{abstract}

\section{Keywords}

Collaborative information retrieval, social information retrieval, task, evaluation

\section{INTRODUCTION}

The paradigm of Collaborative Information Seeking (CIS) and Retrieval (CIR) refers to methodologies and technologies that support collective-knowledge sharing within a work team in order to solve a shared complex problem [5]. Accordingly, the main underlying challenge is to satisfy the mutual beneficial goals of both individual users and the collaborative group while maintaining a reasonable level of cognitive effort underlying users' interactions [11]. Indeed, collaborative search is also known as a social process [4] in which users leverage from other users' interactions and social signals (e.g., bookmarks and annotations). In the recent years, several studies $[7,16,8]$ have been carried out to understand the behavioral process of users in terms of question asking on search engines vs. social networks. In this context, social platforms (e.g., social networks $[9,2]$ and community question-answering $[6,3]$ ) have been acknowledged as a place where users collaborate to solve an information need. This provides numerous opportunities for new and novel research within the field of CIR/CIS where the gap between social and collaborative search can be brought together.

However, the evaluation for CIS/CIR and social \& collaborative IR models are still challenging, as there are a variety of confounding factors such as the multi-user and multilevel contexts, the exploratory aspect of the search through multi-session search activities, the multiplicity of relevance factors, the individual vs. collective value of relevance, the search interfaces supporting the collaborative interactions as well as the social interactions themselves. While, substantial research advances in the evaluation of non-collaborative information retrieval and seeking tasks have been achieved through international evaluation campaigns such as TREC, CLEF and NTIR, to date and to our knowledge, no standardization effort has been achieved for the evaluation of CIS/CIR and social-CIR. During the first ECol Workshop there was strong consensus that there is an important need to investigate the evaluation challenge in CIS/CIR/socialCIR with the aim of creating common evaluation frameworks that would foster the research area.

A first edition of this workshop has been organized at CIKM 2015 in which we discussed about the issue of evaluation in CIS/CIR [14]. The second edition of the ECol Workshop $^{1}[14]$ focuses the discussion and provides concrete contributions in developing such an evaluation framework for the unique challenges in CIS/CIR and social-collaborative IR. To make the workshop active and the participant proactive, we release datasets and tools so as to help researchers contributing to the formalization of evaluation frameworks for challenging collaborative tasks (that we will identify).

Finally, we hope that this workshop will be beneficial for the community in both short- and long-term. First, this would allow to understand relevance factors or design standard evaluation frameworks. Second, provided resources (datasets, tool, and tasks) would remain available for the whole community, facilitating in the future comparable and reproducible experiments.

\section{SCOPE AND NOVELTY OF THE WORKSHOP}

These last years and particularly since 2005, CIS and CIR have became emerging topics that have been addressed in

\footnotetext{
${ }^{1}$ https://www.irit.fr/ECol2015/
} 
several IR and IS conferences including CIKM and SIGIR conferences $[1,14]$. While the potential of collaboration has been highlighted with respect to individual settings, other challenges remain and need to be thoroughly explored. Despite most of experimental evaluations have been done with the objective of highlighting the synergic effect of the proposed contributions, there is an important need for the future to discuss about what should be evaluated in terms of collaboration aspects (e.g. cognitive effort, mutual beneficial goal satisfaction, collective relevance...). Moreover, it does not exist standard framework as proposed in ad-hoc information retrieval through the evaluation campaign, as those proposed by TREC, INEX, CLEF, etc.

While a follow on from our previous workshop, this workshop has two distinguishing and novel elements: (i) it has a specific focus on social IR and collaborative IR evaluation, bridging the gap within this space, and (ii) it provides datasets, tools and new tasks for participants and others to undertake evaluations and explore this space. We believe that formalizing evaluation frameworks for a such domain would lead researchers to investigate this research area and propose new social-collaborative models.

Participants will have different ways to participate to the workshop:

- Use the provided datasets/tools to propose an evaluation framework for the identified tasks.

- Use their own datasets/tools to propose an evaluation framework for the identified tasks.

- Use the provided datasets/tools to propose an evaluation framework for a task they have identified.

- Propose an evaluation framework without support of datasets/tools for a task we/they have identified.

- Designing possible tasks (with/without proposing models and evaluation frameworks) on provided or their own datasets.

This opens future research directions and would lead to the design of evaluation frameworks. This would also enable comparable contributions in terms of evaluation framework and constructive discussions throughout the workshop.

\section{RELEASED DATASETS AND TOOLS}

To make the workshop interactive and also in a long-term objective in the field, we share resources and tools:

- Datasets:

- Social-based datasets: tweet collections about two crises, respectively the Ebola virus epidemic and the hurricane Sandy ${ }^{2}[13,15]$.

- Collaborative search $\operatorname{logs}^{3}[12]$.

- Tools:

- Coagmento collaborative interface [10]

- Open-source version of Coagmento for doing userstudies on github ${ }^{4}$

\footnotetext{
${ }^{2}$ https://figshare.com/collections/expac/3283118

${ }^{3}$ http://infoseeking.org/data.php\#cis2010

${ }^{4}$ https://github.com/InfoSeeking/CoagmentoCollaboratory
}

\section{EXAMPLES OF TOPICS}

Taking into consideration the release of datasets and tools, we will propose different topics that would guide participants in their contributions. For each topic, participants would be invited either to design the task or formalize the evaluation framework (including metrics, datasets, ground truth, baselines, etc.). Below we list examples of topics:

- Recommending social collaborators (experts, answerers, sympathizers)

- Collaborative ranking on social platforms (criteria: federated, novelty, diversity, interactive, time consideration)

- Identifying impacting factors on search effectiveness (failure, success, struggling).

- Preserving data privacy through multi-level CIR.

- Exploratory search (knowledge acquisition, multifaceted search).

- Collaborative intent understanding.

\section{INTENDED PROGRAM COMMITTEE}

- Thilo Boehm, University Hagen - Germany

- Luanne Freund, University of British Columbia - USA

- Martin Halvey, University of Strathclyde - UK

- Preben Hansen, Stockholm University - Sweden

- Ryan Kelly, University of Bath - UK

- Simon Knight, University of Technology Sydney - Australia

- Stephann Makri, University of London - UK

- Jian-Yun Nie, University de Montreal - Canada

- Takehiro Yamamoto, Department of Social Informatics, Graduate School of Informatics, Kyoto University - Japan

\section{REFERENCES}

[1] CIR '11: Proceedings of the 3rd International Workshop on Collaborative Information Retrieval, New York, NY, USA, 2011. ACM. 605110.

[2] D. Castilho, P. Melo, D. Quercia, and F. Benevenuto. Working with friends: Unveiling working affinity features from facebook data. In ICWSM, 2014.

[3] S. Chang and A. Pal. Routing questions for collaborative answering in community question answering. In ASONAM, pages 494-501. ACM, 2013.

[4] B. M. Evans and E. H. Chi. An elaborated model of social search. IPEGM, 46(6):656-678, 2010.

[5] J. Foster. Collaborative information seeking and retrieval. Annual Review of Information Science and Technology, 40(1), 2006.

[6] B. Li and I. King. Routing questions to appropriate answerers in community question answering services. In CIKM. ACM, 2010. 
[7] M. R. Morris, J. Teevan, and K. Panovich. What do people ask their social networks, and why?: A survey study of status messages. In $C H I$, pages $1739-1748$, 2010.

[8] A. Oeldorf-Hirsh, M. R. Morris, J. Teevan, and G. Darren. To search or to ask: The routing of information needs between traditional search engines and social networks. In ICWSM, 2014.

[9] S. A. Paul, L. Hong, and H. Chi. Is twitter a good place for asking questions? a characterization study. In ICWSM, 2011.

[10] C. Shah. Coagmento- a collaborative information seeking, synthesis and sense-making framework. In Integrated demo at CSCW 2010, 2010.

[11] C. Shah and R. González-Ibáñez. Evaluating the synergic effect of collaboration in information seeking. In SIGIR, pages 913-922, 2011.
[12] C. Shah and R. Gonzalez-Ibanez. Collaborative information seeking lab experiments dataset. http://infoseeking.org/data.php\#cis2010, 2017.

[13] L. Soulier, L. Tamine, and G. Nguyen. Answering twitter questions: a model for recommending answerers through social collaboration. In CIKM, pages 267-276, 2016.

[14] L. Soulier, L. Tamine, T. Sakai, L. Azzopardi, and J. Pickens. Report on the first international workshop on the evaluation on collaborative information seeking and retrieval (ecol'2015). SIGIR Forum, 50(1):42-48, 2016.

[15] L. Tamine, L. Soulier, L. Ben Jabeur, F. Amblard, C. Hanachi, G. Hubert, and C. Roth. Social media-based collaborative information access: Analysis of online crisis-related twitter conversations. In $H T$, pages 159-168. ACM, 2016.

[16] J. Teevan, D. Ramage, and M.-R. Morris. Twitter search: A comparison of microblog search and web search. In WSDM, pages 35-44, 2011. 